\title{
Les controverses environnementales : Entre conflit
} et consensus

Jérôme Lafitte

\section{CpenEdition Journals}

Édition électronique

URL : http://journals.openedition.org/ere/453

DOI : $10.4000 /$ ere.453

ISSN : 2561-2271

Éditeur

Centr'ERE

Référence électronique

Jérôme Lafitte, "Les controverses environnementales : Entre conflit et consensus », Éducation relative à l'environnement [En ligne], Volume 12 | 2015, mis en ligne le 20 mai 2015, consulté le 21 février 2020. URL : http://journals.openedition.org/ere/453 ; DOI : 10.4000/ere.453 


\title{
Les controverses
}

\section{environnementales : Entre conflit et consensus}

\author{
Jérôme Lafitte
}

1 La controverse publique s'est imposée dans nos sociétés contemporaines comme une forme du discours démocratique ${ }^{1}$. En effet, aucun pouvoir autoritaire ne peut se permettre de tels débats publics entre une pluralité d'acteurs, car ils offrent justement la possibilité de remettre en question une parole d'autorité. La controverse ouvre un espace public où se déploie une certaine liberté d'expression en confrontant des savoirs et des argumentaires différents. Reste qu'une telle forme de discours public - donnant lieu à des stratégies persuasives qui cherchent à infléchir l'interprétation des récepteurs - n'est pas la panacée d'une démocratie dont elle incarnerait toutes les vertus. En référence à Bourdieu (1980, p. 10), il est possible d'observer en effet qu'elle peut favoriser des stratégies de pouvoir : ainsi une certaine éloquence, en tant que pratique culturelle cherchant à distinguer sa conduite du vulgaire, pourrait viser à attirer des avantages sociaux à ceux qui la maîtrisent. D'autres travaux issus de la sociologie, tels que ceux de Callon (1981), ont montré que les controverses ne sont pas nécessairement démocratiques: certains acteurs d'influence vont déterminer la légitimité des problèmes à aborder, et ce faisant, excluent d'autres acteurs sociaux.

La montée des préoccupations environnementales a favorisé le croisement de savoirs rattachés à différents domaines de connaissance, qu'il s'agisse d'expertises scientifiques ou juridiques en relation avec la santé ou les technologies, mais également d'expertises dites profanes, émanant de la société civile. Les problématiques environnementales ont cela de spécifique que leurs effets se manifestent au sein des territoires, dont les acteurs-habitants sont experts de ces milieux de vie. La dimension sociotechnique des problématiques environnementales démultiplie la diversité des intérêts des parties prenantes et de leurs agendas, accentue la vulnérabilité des habitants des territoires concernés et la complexité des enjeux, et rend la clôture d'une controverse environnementale d'autant plus délicate qu'elle est arrimée à des mouvements sociaux. 
Plusieurs défis d'ordre démocratique mais aussi scientifique surgissent, que la recherche en éducation peut contribuer à éclairer.

3 Cet article envisage la controverse comme une forme discursive rémanente de la construction du savoir. La controverse repose sur une pratique sociale de discussion d'un problème qui appelle la confrontation d'une diversité de savoirs entraînant la construction d'un savoir actualisé. Cette pratique discursive1 s'est progressivement instituée au fil de l'histoire au point de devenir une forme incontournable, notamment dans le cas de problématiques environnementales. Cependant, chaque controverse reste un évènement discursif singulier à étudier en contexte.

Notre proposition est que dans la dynamique d'une controverse environnementale, la construction du savoir alterne entre deux modalités discursives: la recherche de consensus et l'usage du conflit. La situation de controverse en tant que pratique d'échange et de production d'énoncés dans un contexte déterminé est marquée par une dialectique fonctionnelle entre polémique et accord formant deux figures discursives repérables qui sont opposées et complémentaires, la figure de la discorde axée sur l'usage du conflit, et la figure de la concorde axée sur la recherche de consensus. Nous tenterons de mettre à l'épreuve le bien-fondé et la portée de la controverse ainsi envisagée dans le champ de l'éducation environnementale en insistant davantage sur le domaine de recherche plus spécifique de l'éducation des adultes relative à l'environnement (EAE)².

La première partie de cet article esquissera la généalogie de la controverse en menant une analyse interprétative, conceptuelle et historique de cette pratique discursive qui donne lieu à une construction de savoirs, à partir des premières préoccupations philosophiques d'une éducation au raisonnement critique jusqu'à son rôle actuel dans la diffusion publique des problématiques environnementales. Pour ce faire, nous tenterons de montrer comment les deux figures proposées de la discorde et de la concorde sont identifiables et peuvent contribuer à la compréhension des controverses environnementales actuelles dans une perspective éducative. La seconde partie orientera la réflexion sur la place et l'usage de la controverse environnementale dans le champ de l'EAE. Elle sera centrée sur l'éducation non-formelle et informelle et s'appuiera sur une recension d'écrits récents visant à circonscrire la place de cet objet en EAE et l'usage qui est et qui pourrait être fait des deux figures associées de la discorde et de la concorde.

\section{Ébauche d'une généalogie de la controverse ${ }^{3}$}

\section{Première théorisation gréco-romaine}

La controverse est une pratique discursive ancienne de production du savoir. Durant l'Antiquité, plusieurs penseurs grecs défendent l'idée qu'à chaque argument, un contreargument d'égale valeur est possible (Gil, s. d.). La discorde (diaphonia) apparaît alors comme l'un des motifs de la suspension du jugement durant l'échange d'arguments. L'état de tranquillité est nécessaire pour examiner les relations entre les moyens l'argumentation - avec les fins - la question de la relation à la vérité. Il s'agit de pouvoir juger « en connaissance », avec la sérénité qui convient à la prise de décision réfléchie. Pour les Sceptiques, l'une des premières tournures argumentatives est le désaccord. Une telle attitude argumentative ouvre sur deux postures: la première est qu'il convient de tenir compte de la force de tout argument par les effets produits, par 
exemple en termes d'émotions, et pas seulement la force en soi de l'argument; la seconde interdit de prendre toute affirmation gratuite pour une vérité. La Rome antique articule plutôt le couple controversia/disputatio. Selon Fabiani (1997), la controversia renvoie au conflit qui se règle par le duel judiciaire à des fins de décision, tandis que la disputatio est plutôt réservée aux discussions philosophiques sans litiges.

Cette première théorisation de la controverse est d'une grande portée, puisque les principes énoncés recoupent les notions de vérité, de certitude, de jugement en vue de la décision. La suspension du jugement est une étape qui fonde la prise de décision, cette dernière reposant sur la force des arguments et des savoirs mobilisés à cet effet.

La controverse est donc bien une pratique de référence ancienne pour la pensée critique et le dialogue des savoirs. Le scepticisme scientifique ou doute méthodique qui s'appuie sur l'esprit critique et la méthode scientifique autour de la réfutabilité des théories et de la répétabilité des expériences - a depuis longtemps relayé la posture philosophique des penseurs gréco-romains. Dans Le Sophiste (p. 231 d, cité dans Gil, s. d.), Platon ne posait-il pas le dialogue critique comme une voie de "purification de l'âme des opinions qui font obstacles aux sciences"? On retrouve ici la «fonction ordalique" du conflit, sorte d'interrogation du destin pour en finir avec les spéculations et l'incertitude angoissante d'une situation bloquée et ainsi, renouer avec l'action (Monroy, 2004, p. 31).

\section{Vers une esthétisation de la figure de la controverse}

9 La Renaissance fait de la controverse un art de la discussion à part entière. La période est marquée par la publication du Traité des Controverses, rédigé par le cardinal Bellarmin. Devenu maître dans l'art de la controverse religieuse, il se serait distingué par sa compétence théologique mise au service de la foi catholique, mais également par sa courtoisie vis-à-vis des protestants, à une époque où les débats tournaient vite aux injures et aux attaques personnelles. La controverse devient un art dans lequel le respect des personnes ne cède en rien à la réfutation des doctrines erronées. Une telle règle demeure un fondement éthique de la controverse scientifique. En effet, celle-ci s'établit sur le principe de la réfutation des arguments et non sur les attaques personnelles, qui discréditent toute argumentation.

\section{Controverses et raison critique}

Durant la période moderne, la controverse devient dans l'opinion, «comme une malédiction de la pensée et l'indice de la fragilité du discours philosophique » (Gil, s. d.). Ce fut la position d'un Bacon ou d'un Leibniz pour qui le « calcul», devait constituer la façon de mettre fin aux controverses. Une telle posture est également au fondement de la philosophie kantienne à travers la volonté d'arrêter les « combats sans fin » de la métaphysique. Mais une seconde attitude existe chez Kant à l'égard des controverses, celle qui porte intérêt au conflit de la raison avec elle-même, une praxis dévoilant les limites internes de la pensée et réprimant les extravagances de la raison.

Durant cette période, l'idée de controverse se consolide donc autour de deux modalités discursives particulières qui mobilisent l'usage de la raison, le conflit et le consensus, qui correspondent à des figures discursives repérables dans toute controverse. 
12 La figure de la concorde s'affirme par la confiance dans la puissance de la raison pour parvenir à un consensus entre positions rivales. Une position conflictuelle est assimilée à un indice de la fragilité du discours. Le conflit est une malédiction de la pensée, un déficit de raison à corriger par la recherche de l'harmonie des points de vue. En creux, le conflit apparait comme négatif, stérile ou à tout le moins risqué. Mais la recherche de consensus à tout prix peut s'avérer tout autant stérile pour la construction et l'échange de savoirs en vue de l'amélioration de la situation, ici socioécologique.

La seconde figure discursive, plus dialectique, se nourrit du conflit pour clarifier des positions, ceci grâce à la confrontation des raisonnements et des savoirs, en laissant ouvertes les opportunités de définition de l'objet même de la controverse. La mise en œuvre d'une réflexivité à l'égard de la raison elle-même, permet de se "penser pensant " et de situer les savoirs qui émergent et les connaissances mobilisées, en prêtant attention au contexte. Pour penser «sur » un sujet, il peut être utile de penser " contre ", de manière dialectique grâce à une mise à l'épreuve critique des savoirs qui se construisent dans l'interaction sociale et dans les débats que l'on mène au sein de sa propre pensée. Il s'agit de la figure de la discorde, mais cette appellation ne porte pas ici en elle-même de jugement de valeur péjoratif. Certes, la radicalisation des positions peut bloquer la situation et limiter la construction et l'échange de savoirs, mais la discorde peut aussi être féconde.

Figure 1 : Les deux figures discursives de la controverse

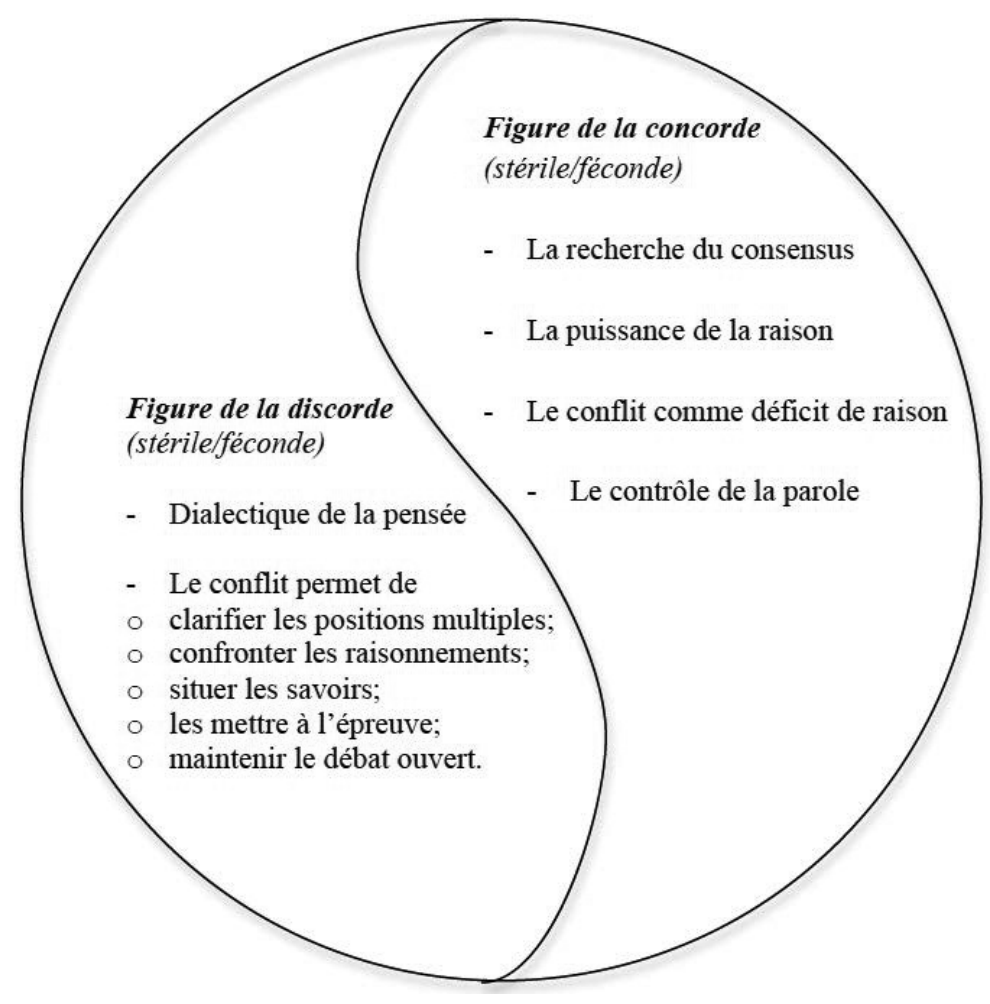

14 Ainsi, la pratique discursive de la controverse donne lieu à un échange de savoirs qui peut prendre une forme consensuelle ou conflictuelle, correspondant à deux modalités discursives dialectiques et complémentaires : la figure de la discorde et la figure de la concorde. Ces deux figures peuvent être à leur tour déclinées selon leur effet, stérile ou fécond, en termes de participation des parties prenantes et des citoyens concernés, 
mais aussi au regard du dialogue des savoirs susceptible de contribuer à la coconstruction de nouveaux savoirs éclairant les enjeux de la controverse. En matière d'environnement, il s'agit de favoriser une prise de décision qui tienne compte des principes de précaution et de responsabilité (Figure 2), mais également d'un principe de reconnaissance de la légitimité des savoirs citoyens.

\section{Des controverses scientifiques aux controverses sociotechniques}

Les controverses ont toujours fait partie de l'avancée des sciences et sont même indispensables à la formation, au développement et à l'évaluation des théories, des méthodes, de la constitution et de l'interprétation des données. La science est souvent considérée comme une succession ininterrompue de controverses qui perdurent ou sont reformulées. Les controverses scientifiques mettent en jeu des oppositions catégoriales fondatrices, indispensables à la compréhension des phénomènes scientifiques. Ces oppositions agissent comme autant de contraintes cognitives qui sont l'occasion de stratégies discursives et cognitives: par exemple, en sciences de l'environnement, l'opposition entre le paradigme de l'impact et celui de la vulnérabilité, ou en éducation, entre le paradigme de l'enseignement et celui de l'apprentissage.

De nos jours, les questionnements environnementaux semblent avoir renforcé le développement des controverses technoscientifiques qui saisissent le savoir comme étant d'emblée une connaissance "publique ", partagée et divisée. Greffées autour de technologies susceptibles de causer des dommages graves pour l'environnement voire les générations futures, les controverses sociotechniques concernent directement le quotidien des usagers et leurs territoires. Au sein du public, les objets scientifiques s'y présentent réfractés par les interactions sociales, mais aussi par la médiatisation de telles controverses ; les oppositions théoriques sont psychiquement investies (Gil, s.d.). Pour le chercheur qui les étudie, de telles controverses sont un observatoire privilégié de l'entreprise scientifique. Elles donnent à voir le processus de production et de circulation du savoir à partir de la diversité des protagonistes qui s'appuient sur des postures et des « paradigmes » différents ou opposés. Elles témoignent d'enjeux relatifs aux logiques concurrentielles entre laboratoires, chercheurs, cabinets d'expertise, qu'il convient de contextualiser socialement, pour saisir pleinement les positionnements et la mise en ordre du discours qui survient dès la formulation des problèmes (Callon, 1981).

\section{Vers une complexification de la lisibilité des controverses environnementales}

17 La dimension très appliquée et instrumentale des technosciences, leur ancrage dans les territoires de vie, ainsi que l'horizon public des controverses environnementales créent en effet une certaine rupture avec la figure de la controverse scientifique. Dans un rapport sur les controverses environnementales au Québec, Limoges et coll. (1993, p. 57) ${ }^{4}$ rappellent que le modèle scolastique de la disputatio sans litiges de la controverse scientifique ne correspond plus à la réalité des controverses publiques environnementales. 
18 Il n'y a plus une seule question en jeu avec deux parties : ceux qui supportent une option et les opposants. Les controverses environnementales sont désormais polycentrées et multiréférentielles par la diversité des domaines disciplinaires et des univers de pertinence des acteurs concernés. La Rochelle et Désautels (2006) insistent sur les rétroactions du social sur la recherche, via le développement des expertises profanes souvent assumées par des mouvements sociaux et mises en valeur par les travaux socio-anthropologiques de Callon, Lascoumes et Barthe (2001), entre autres. Ces auteurs montrent que les procédures authentiquement participatives ainsi qu'une éducation critique du public constituent des outils uniques, à la hauteur de l'exigence démocratique et des pouvoirs grandissant de la technoscience.

Dans la controverse environnementale publique, il ne s'agit pas d'établir la vérité : l'horizon de telles controverses est celui de la prise de décision qui ne peut s'opérer qu'avec de grandes marges d'incertitude. Les échanges de savoirs se complexifient autour de la remise en cause de l'ancienne "démarcation" ou "coupure épistémologique " nécessaire (Bachelard) entre science et non science (Popper). La nature positiviste de cette démarcation rejette les éléments psychosociologiques ou les conjonctures historiques à l'extérieur de l'espace du débat, espace alors déshistoricisé et cantonné à un ordre logique, selon Fabiani (1997). La façon dont la controverse va se dérouler (processus) - notamment l'interaction évolutive entre acteurs (dynamique) est alors déterminante dans la perspective d'une prise de décision que l'on voudrait démocratique et participative.

\section{Des stratégies à l'œuvre au sein des controverses}

La pratique discursive de la controverse s'inscrit dans un des domaines de connaissance et d'analyse des discours les plus anciens qui soient, la rhétorique qui est l'action du discours sur les esprits, un art de persuader mais aussi de « bien dire ». Tout discours, à plus forte raison intervenant dans le contexte d'énonciation du débat public, met en relation une pluralité d'acteurs. Ces derniers portent des compétences énonciatrices diverses, des intérêts et des visions du monde parfois contradictoires, qui peuvent rendre la construction du sens ainsi que la mobilisation et l'échange de savoirs à cet effet pour le moins polyphonique. Dans cet ordre d'idée, considérant les dynamiques de savoir et les logiques de pouvoir qui s'affrontent au cours de l'évolution d'une controverse, la notion de figure de discours peut être rapprochée de l'idée de "masque $»^{5}$, soit une stratégie d'influence de l'opinion publique. La situation de controverse donne à voir une construction du savoir mais aussi une mise en ordre du discours, ce dernier devenant l'écho des logiques de pouvoir et des dynamiques de savoir comme le montrent les travaux de Foucault (1969).

Limoges et coll. (1993) précisent que les controverses ne sont pas nécessairement l'indice d'une carence, le signe d'un échec politique ou administratif mais plutôt des moments privilégiés d'expression et d'explicitation des préoccupations publiques. Le rapport invite les gestionnaires du processus à renforcer sa qualité comme garantie de la crédibilité donc, de la possibilité d'une clôture robuste et consensuelle de la controverse. En effet, la parole et l'argumentaire des citoyens-usagers concernés par une situation de controverse environnementale sont souvent dénigrés sur les scènes publiques et médiatiques, et réduits à des attitudes irrationnelles, émotives et injustifiées, signes d'une carence en information ou d'un manque d'ouverture (Batellier 
et Sauvé, 2011; Blondiaux, 2008). Les controverses environnementales révèlent une mise en ordre du discours parfois violente, par des logiques de pouvoirs qui mettent en œuvre des "pratiques divisantes" concernant les connaissances échangées, notamment la division entre connaissances « légitimes » et connaissances délégitimées parce qu' «irrationnelles». Celles-ci se trouvent alors exclues du régime de vérité circonstanciel à telle controverse environnementale. La configuration d'une controverse environnementale donne à voir la construction d'un espace social relationnel et concurrentiel, véritable champ de forces où s'accumulent et circulent des savoirs et des pouvoirs, distribuant de manière sélective un ensemble de positionnements tenus par les acteurs impliqués dans cette controverse. La figure 2 tente de rendre compte très schématiquement d'un tel champ de forces.

Figure 2 : Champs de forces et controverses environnementales

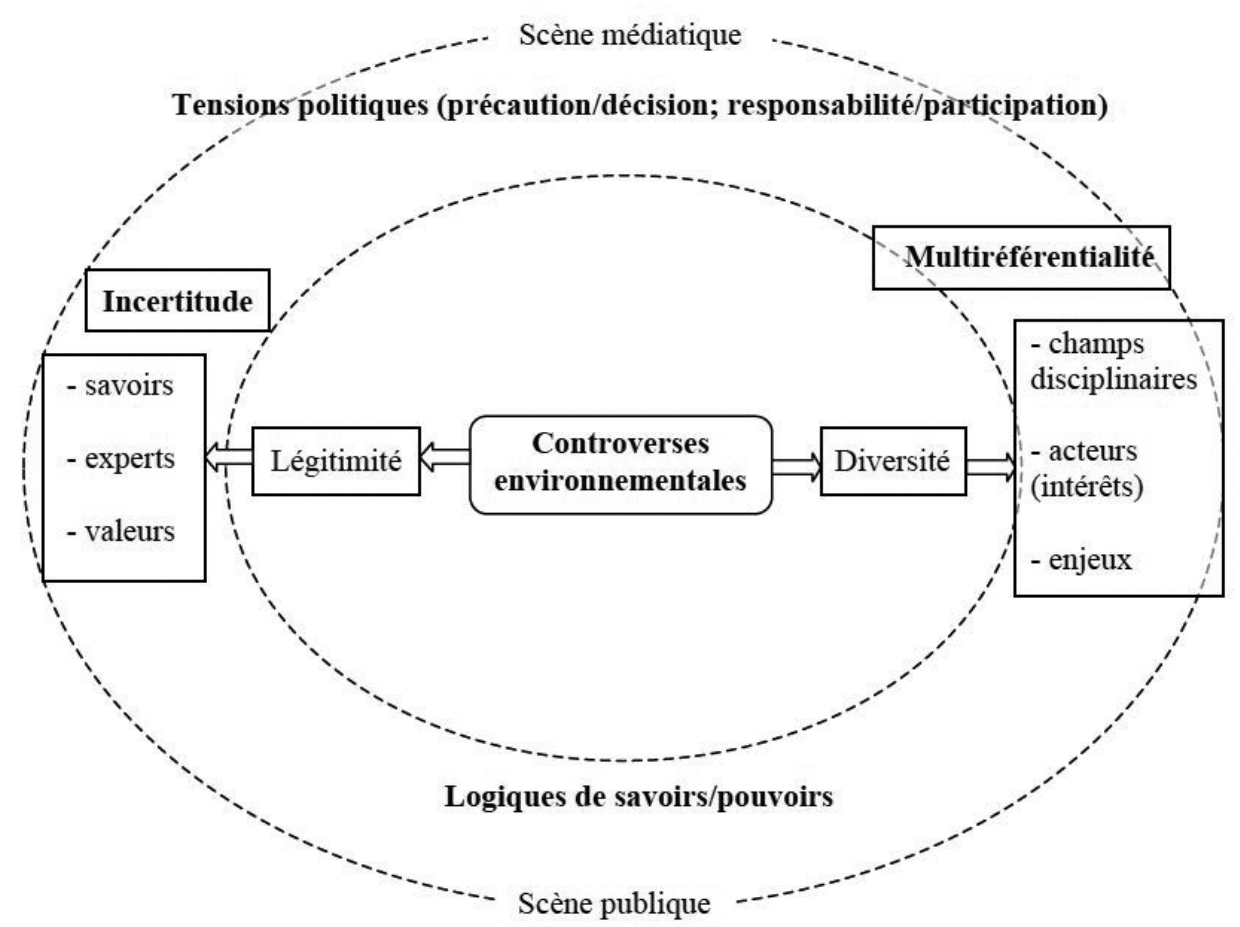

Dans le même temps, la parole des experts peut être instrumentalisée par les parties prenantes de la controverse environnementale au service de leurs intérêts propres, contribuant à une certaine désinformation. Comme le montre une étude de Yannick, Kervarec et Verena, (2009 p. 2), "pour les "acteurs forts" tels l'État, les propriétaires fonciers ou les promoteurs de projets d'intérêts publics, la concertation constitue une manière d'éviter le conflit (et intrinsèquement, un règlement par un tiers qui leur échapperait) ». Pour d'autres et notamment les « acteurs faibles », elle peut constituer une stratégie pour mobiliser les acteurs du territoire afin qu'ils s'engagent dans la concertation.

Un tel clivage recoupe le débat actuel entre les tenants de la théorie de la démocratie délibérative et les tenants de la théorie de la démocratie agonistique (Blondiaux, 2008). Ces derniers reprochent aux premiers de faire reposer la légitimité du processus décisionnel sur l'échange d'arguments rationnels visant le consensus. Cette procédure quasi exclusive et illusoire mènerait à une décision légitime. Or, cela se ferait au prix d'une double exclusion. La première concerne les groupes dominés de citoyens, 
considérés comme minorités par la majorité et parfois même "déviants ». La seconde exclusion concerne le conflit lui-même, réduit à une simple opposition d'opinions. Les tenants de la démocratie agonistique prêtent au conflit une dynamique plus constructive et créatrice. La lutte dans laquelle entrent les groupes dominés se fait sur la définition même des questions posées et la vision du monde concernée. Les tenants de la démocratie agonistique insistent par ailleurs sur le caractère irréductible du conflit qui mobilise des identités d'acteurs plurielles. Au final, ces derniers ne se mettent que très rarement d'accord et gardent des conceptions opposées de la meilleure solution. Cette contextualité de l'affrontement et des rapports de pouvoirs dans la controverse joue un rôle essentiel dans la création d'identités politiques multiples. En effet, le politique porte autant sur la capacité à faire éclater les cadres habituels des procédures normatives qui font société, que sur l'acte éminemment social de "poser un problème » politiquement au sein de dispositifs démocratiques tels que les démarches participatives avec leurs normes instituées.

Par ailleurs, il semble que la pratique discursive de la controverse appliquée aux problématiques environnementales en contexte technoscientifique ait brouillé quelque peu la forme discursive de la controverse envisagée selon la vision classique et idéalisée du débat scientifique; mais in fine, on retrouve les deux figures discursives particulières de la discorde et de la concorde. Au-delà des subtilités des solutions régulatrices, Limoges et coll. (1993, p. 3) affirment que "sa gestion demeurera toujours un art ». La controverse publique environnementale reste inscrite dans une continuité historique et épistémique autour des deux figures discursives proposées dans cet article, mais celles-ci sont encadrées par les procédures de régulation publique à des fins de clôture décisionnelle. Or une telle clôture est loin d'être garantie. Par ailleurs, elle est souvent l'occasion d'imposer des décisions irréversibles qui ne sont plus négociables et s'accompagne de l'exclusion durable des citoyens au profit des spécialistes et de technocrates victorieux (Callon, 1981).

L'analyse d'une controverse environnementale envisagée à partir d'une succession de figures discursives, tantôt polarisée par l'harmonie des positions, tantôt par le conflit, nous semble donc éclairante pour saisir les échanges et la construction de savoirs qui caractérisent les dynamiques discursives. Il convient d'apprécier maintenant la potentialité pédagogique de telles figures.

\section{Les controverses environnementales comme situation éducative en éducation des adultes relative à l'environnement}

\section{La controverse environnementale dans la littérature en EAE}

Une recension d'écrits récents a permis de repérer l'objet de la controverse environnementale dans la littérature de recherche en $\mathrm{EAE}^{6}$. Notre recension s'est concentrée sur des revues scientifiques anglophones et francophones, pour des raisons de contraintes temporelles, en limitant cette prospection aux cinq dernières années (2009 incluse). Au-delà de l'objectif principal mentionné, il s'agissait d'identifier si les deux figures de la discorde et de la concorde et leurs deux versants, stérile ou fécond, étaient repérables dans la façon dont les controverses environnementales étaient abordées en EAE. 
27 Cette démarche a permis de constater que la littérature de recherche sur les controverses environnementales est relativement récente et abondante, notamment dans le monde francophone. Pour preuve, le dernier numéro de la revue électronique des sciences de l'environnement Vertigo, portait sur les «Controverses environnementales : expertise et expertise de l'expertise ». Ce numéro de Vertigo est représentatif de l'idée soutenue par de nombreux auteurs qui considèrent l'éducation ou l'apprentissage comme dynamiques fondamentales pour comprendre et participer à de telles controverses afin de peser sur la prise de décision. Cependant, d'une part ces dynamiques sont abordées de façon très générale et d'autre part, la littérature en sciences de l'environnement, plus spécifiquement en sciences politiques ou en sciences sociales ne fait que très rarement référence à l'EAE. Ce champ de savoirs et de pratiques en émergence reste par ailleurs marqué par la grande diversité d'origine de ses chercheurs et n'a pas encore une grande visibilité scientifique. Les chercheurs continuent de publier dans leur discipline de formation et non pas dans la littérature spécifique à l'éducation des adultes, ce qui tend à limiter la diffusion de la recherche et des écrits scientifiques de ce domaine (Fejes et Nicoll, 2013).

Notre recension a permis de confirmer que la controverse environnementale est envisagée comme situation éducative seulement dans le domaine de l'éducation formelle des adultes ${ }^{7}$. Mais pour ce faire, les chercheurs mènent assez fréquemment leurs investigations en considérant à la fois l'éducation au secondaire et l'éducation universitaire, sans que l'on puisse dégager clairement une prise en compte de la spécificité de l'apprenant adulte; ici, les conditions du milieu éducatif priment sur la spécificité de l'apprenant. Pour ces raisons et afin de limiter le propos, les réflexions menées sur ces deux niveaux - secondaire et universitaire - ont été écartées de l'analyse qui fait l'objet de cet article. Par ailleurs, l'EAE est davantage campée dans l'éducation non-formelle et informelle (Villemagne, 2008). Or, dans ces deux contextes, force est de constater qu'aucun article n'aborde la question des controverses environnementales comme situation éducative, et cela aussi bien dans le monde francophone qu'anglophone. Au sujet d'une telle absence, on peut avancer la remarque suivante pour ouvrir la réflexion: la controverse environnementale intervient nécessairement dans le monde adulte aux prises avec des contingences territoriales et socioécologiques. L'urgence liée à la situation de controverse et les tensions socioécologiques qui se cristallisent localement rendent difficiles la pratique de la recherche en éducation (avec sa propre temporalité), aussi bien que l'intervention éducative elle-même, ce que confirme l'un des articles (Golding et coll., 2009). Paradoxalement, le besoin d'éducation, d'information, de formation fait souvent consensus dans les recherches abordant la controverse environnementale, sans que les acteurs concernés, dont les chercheurs, ne différencient de tels processus. À mon sens, cela rend les besoins de recherche spécifique en EAE d'autant plus urgents. Nous nous appuierons donc principalement sur trois articles anglophones qui sont ressortis de cette recension d'écrits centrée sur l'EAE non-formelle et informelle par le fait qu'ils abordent explicitement la controverse environnementale ou proposent un contexte de controverse environnementale. L'absence d'articles francophones confirme la remarque de Fejes et Nicoll (2013) qui déplorent le fait que cette situation limite la diffusion des travaux de recherche en éducation des adultes. 


\section{Brève analyse du corpus d'articles} dynamiques participatives qui se déploient au sein des territoires de vie des acteurshabitants, d'autant que l'enjeu de l'implication des apprenants adultes est majeur en EAE. Compte tenu de la spécificité de l'éducabilité adulte, leur participation comme leur implication dans les activités éducatives restent déterminantes pour renforcer leur pouvoir-agir. De telles dynamiques interviennent principalement en situation nonformelle ou informelle. L'article de Golding et coll. (2009) concerne plus spécifiquement l'éducation non formelle auprès des adultes. L'article de Walter (2012) renvoie davantage à l'éducation informelle tandis que l'article de Bray (2010) cible l'éducation au développement durable sans qu'il soit possible de le positionner dans un contexte éducatif clairement identifié.

Le cadre référentiel du développement durable s'est rapidement diffusé dans le domaine de l'éducation, notamment en contexte d'éducation formelle. L'article de Bray (2010) adopte une posture critique au sujet de la littérature scientifique propre à l'éducation en vue du développement durable (Education for Sustainable Development). Il précise qu'une telle littérature est pleine d'idéaux et d'espoir d'un monde meilleur, se revendiquant de valeurs positives, optimistes et démocratiques. Il en ressort que l'éducation en vue du développement durable (EDD) est présentée comme étant clairement une « bonne chose » selon Bray (2010), qui considère qu'une telle littérature reste marquée par un déficit critique. Or, selon cet auteur, les questions environnementales témoignent de la prégnance des sujets controversés dont les dilemmes socioécologiques constituent un défi de taille, rarement relevé par l'EDD. Le refus de traiter ces controverses environnementales essentielles, par crainte de s'écarter de la science objective et de prendre en compte les valeurs, opinions et croyances, ou par peur du conflit, ou encore par complicité avec le maintien d'un statu quo insoutenable ou non durable, aboutira selon cet auteur, à un échec pour préparer les générations futures à affronter de telles questions. Cet article témoigne des risques que fait courir une certaine mise en ordre du discours du développement durable appliqué à l'éducation si les conditions d'énonciation d'un tel discours persistent à exclure non seulement la pratique discursive de la controverse environnementale, mais aussi les dilemmes socioécologiques du développement durable lui-même. De telles pratiques discursives divisantes et excluantes s'appuient sur des normes et des procédures apparemment démocratiques qui contrôlent voire anesthésient le débat et sélectionnent les «bonnes » connaissances au service d'une "volonté de vérité » qui fait courir le risque de privilégier une conception vertueuse des relations science/ technologies, par souci de légitimation et d'objectivation du discours du développement durable appliqué à l'éducation. La figure discursive de la concorde domine. Pourtant, la mobilisation de la figure de la discorde pour un usage éducatif s'impose, en raison de sa fonction constructive dans la controverse environnementale. Un tel usage est d'autant plus nécessaire dans le champ de l'EDD selon Bray (2010) que le déficit critique existe non seulement sur le plan épistémologique, autour du concept même de développement durable, mais également sur le plan pédagogique, compte tenu des limites d'un apprentissage centré sur l'acquisition de gestes déclinés de manière prescriptive (Clover, 2003 ; Bachelart, 2006 ; Sauvé, 2007 ; Breiting, 2009). 
31 Dans le courant de l'EAE, plusieurs chercheurs privilégient l'étude de l'apprentissage dans les mouvements sociaux, ce qui permet de porter attention au rôle joué par l'implication ou l'engagement dans l'acquisition ou la construction de savoirs (Clover, 2003 ; Walter, 2009). L'article de Walter (2012) présente l'étude de trois cas de situations éducatives informelles au sein desquelles l'identité collective se construit grâce au développement de discours et de prises de positions contestataires au sein des mouvements sociaux. Une telle approche s'inscrit dans la tendance actuelle des chercheurs en éducation des adultes privilégiant la pédagogie critique, les théorisations post-structuralistes ou des approches centrées sur l'apprentissage contextualisé selon des perspectives socioculturelles. Un des enjeux éducatifs porte sur le processus d'apprentissage, plus spécifiquement sur l'articulation entre une mobilisation de savoirs sur le court-terme voire dans l'urgence, et la nécessité d'une mobilisation de tels savoirs environnementaux à long terme. Cette préoccupation n'est pas nouvelle dans les recherches qualitatives actuelles qui ne se satisfont plus d'indicateurs formels, limités à un geste, une observation à un moment donné. Dans les trois cas étudiés par Walter, les personnes engagées jouent le rôle d'opposant et de catalyseur d'un contrediscours. Elles suscitent ainsi l'organisation d'un réseau d'acteurs par des actions créatives et symboliques qui proposent au public de nouvelles grilles de lecture. Elles libèrent ainsi des espaces de discussion et de remise en question des cadres et discours hégémoniques, avec leurs codes de communication et de compréhension aliénants. Par leur créativité et leur implication pérenne, de telles personnes favorisent la recomposition des identités collectives et individuelles - politiques et écologiques, c'est-à-dire environnementales (Thomashow, 1995) - des acteurs impliqués et des groupes sociaux interpelés. Ces dynamiques éducatives informelles s'appuient sur la figure discursive de la discorde comme entrée dans les controverses environnementales. Ces cas rendent comptent d'outils puissants pour catalyser la conscientisation à l'égard des questions environnementales, articulant préoccupations locales et globales. Cet "activisme éducatif » renforce le pouvoir-agir des acteurs adultes mobilisés. Les trois situations fortement conflictuelles analysées dans l'article ont fait évoluer les situations, notamment sur le plan politique, jusqu'à générer des changements profonds en faveur d'une amélioration des relations aux réalités socioécologiques locales, aussi bien au niveau personnel que collectif.

L'article de Golding et coll., (2009) évoque un cadre d'analyse très proche des caractéristiques associées aux controverses environnementales. Le cadre d'analyse dit des "problèmes pernicieux» (wicked problems) est constitué de plusieurs caractéristiques. On y retrouve la multidimensionnalité ou l'interconnexion des problèmes qui rend difficile de les étudier séparément ; l'incertitude scientifique associée au fait que toute solution technique à de tels problèmes ouvre sur de nouveaux problèmes ; les conflits de valeurs et de compréhension qui sous-tendent ces problèmes et sont à l'origine de paradoxes que la science peut informer sans pour autant résoudre, donnant lieu à des négociations, sans qu'aucune solution claire n'émerge ; la méfiance (mistrust) entre les parties prenantes; et enfin l'urgence qui implique de tenir compte de solutions efficientes et créatives appropriées aux acteurs locaux qui vivent les problèmes environnementaux sur le terrain. En effet, les problèmes envisagés ne peuvent être résolus par l'accumulation et l'application de connaissances sans tenir compte de la sagesse populaire. 

intervenant en Australie dans un contexte de sécheresse à répétition. Les auteurs explorent les potentialités d'apprentissage pour favoriser une adaptation des communautés face à de tels problèmes. L'article s'inscrit dans l'approche de l'apprentissage contextualisé selon des perspectives socioculturelles. Les analyses produites par la collaboration entre chercheurs et acteurs locaux mettent au jour plusieurs conflits de valeurs et de compréhension entre parties prenantes concernant l'usage de l'eau, la représentation de la situation ou encore le processus décisionnel luimême. Les discours et les postures d'experts, des gouvernements ou encore d'autres communautés locales sont mis en cause. La conclusion recommande la mise en œuvre d'une " atmosphère d'interconnexion " au service d'une " communauté de pratique où les connaissances et l'apprentissage sont partagés parmi toutes les parties prenantes » (p.564, trad. lib.). termes de «capital social » domine. Cela atteste de la difficulté pour ces chercheurs en contexte interdisciplinaire, d'ancrer résolument leur discours dans un paradigme éducatif clair qui aurait pu davantage profiter des potentialités éducatives de la figure de la discorde. Celle-ci est en effet bien présente dans l'étude, par l'entremise du cadre d'analyse dit des "problèmes pernicieux ». Mais l'analyse du conflit des valeurs et de compréhension est ici limitée à une fonction descriptive et analytique qui semble peu favorable à l'action. Certains acteurs manquent en effet de confiance à l'égard de l'efficacité des solutions éducatives issues des organismes gouvernementaux et des experts auprès des communautés d'habitants et fermiers. Certaines communautés locales se méfient par ailleurs des chercheurs qui recueillent des données sans collaborer en termes de solutions notamment. Par ailleurs, le diagnostic résultant de la partie évaluative de l'étude de Golding et coll. (2009) montre plutôt des apprentissages de type comportementaliste, ainsi que des connaissances factuelles et organisationnelles peu partagées et fragmentées entre les communautés étudiées et donc très peu intégrées ne serait-ce qu'à l'échelle d'un bassin-versant. Or, face à l'ampleur des défis écologiques, un des écueils souvent pointé par les recherches en éducation environnementale au sujet de dynamiques éducatives limitées à ce type de connaissances ou d'apprentissage est le risque d'un sentiment d'impuissance («learned hopelessness»), souvent renforcé par un manque d'intégration des savoirs des apprenants au processus éducatif et un manque d'implication des éducateurs ou chercheurs impliqués (Welsh, 1993 ; Villemagne, 2008 ; Breiting, 2009).

L'article montre que certains acteurs locaux ont tendance à focaliser sur les aspects négatifs des positions prises par les autorités politiques, les experts et les environnementalistes qu'ils dénoncent. Une défiance s'installe et cristallise, de façon quelque peu réductrice, le ressentiment envers de telles instances de savoir et de pouvoir. La figure de la discorde se trouve ici renforcée. Mais elle se réduit à des dichotomies stériles qui ne favorisent pas l'implication des acteurs locaux dans la construction et l'échange de savoirs, et qui sont peu constructives à long terme en vue de la résolution de la controverse environnementale (Monroy, 2004 ; Yannick et coll., 2009). La visée exploratoire et diagnostique en termes d'apprentissage, de même que le temps limité de l'étude de Golding et coll. (2009) entravent sans doute la construction d'alternatives éducatives plus ancrées dans une pédagogie critique qui aurait pu s'appuyer de façon constructive et éducative sur la figure de la discorde. Les données

Éducation relative à l'environnement, Volume 12 | 2015 
collectées dans le cadre d'analyse dit des " problèmes pernicieux " pouvaient pourtant le laisser supposer. Une posture critique impliquant les acteurs locaux dans la controverse environnementale pourrait favoriser un approfondissement de la compréhension des logiques de pouvoir et de savoir qui structurent la mise en ordre du discours et les jeux d'intérêt, au-delà des connaissances environnementales nécessaires et complexes à acquérir. Mais une telle approche s'inscrit souvent dans un long terme. Par conséquent, elle reste difficile à planifier et se heurte aux impératifs d'une prise de décision à court terme et ici, de l'urgence d'une adaptation à des épisodes de sécheresse.

\section{Discussion à partir de la comparaison des articles}

Il ressort de l'analyse des articles retenus plusieurs points communs malgré des contextes éducatifs très différents. Dans tous les cas présentés, il s'agit de transformer la relation entre les individus, les sociétés et les réalités socioécologiques au regard des problèmes environnementaux. Dans une telle perspective, les approches exclusivement basées sur les connaissances factuelles et le changement de comportement sont dénoncées comme pauvres en termes d'apprentissage et inefficaces dans la perspective des changements espérés. Or, malgré la volonté affichée d'un apprentissage porteur de changements, ces derniers restent difficiles à mettre en œuvre devant la complexité des situations collectives et individuelles interreliées et ancrées dans les contingences contextuelles territoriales, avec leurs singularités socioéconomiques ou politicoculturelles.

Un premier commentaire concerne la question de l'articulation de la diversité des temporalités en jeu. L'une des temporalités à prendre en considération est celle du temps nécessaire pour la mise en œuvre d'approches pédagogiques plus émancipatrices et critiques, mais exigeantes en termes de maturation. Il s'agit ici de profiter des situations de controverses environnementales pour faire l'examen critique des argumentaires en proposant des activités, des mises en situation, mobilisant successivement les deux modalités discursives de la controverse environnementale. Une telle approche historicisée pourrait prendre en compte deux plans de contextualisation: la logique du débat au niveau micro de la controverse environnementale et son ancrage local, et au niveau macro, «l'horizon du questionnable » qui renvoie aux «stocks d'énoncés disponibles à un moment donné de l'histoire » (Fabiani, 1997, p. 11). La logique du débat commence par le conflit dont l'analyse peut alors s'appuyer sur les figures discursives de la discorde et de la concorde dans leurs deux versants (stérile et fécond) afin de reconstituer les étapes du débat à travers l'enchaînement des arguments et des raisonnements qui se confrontent ou se recoupent en mobilisant des savoirs souvent de manière dialectique ou encore, recherchant un consensus. Cela permet de clarifier les multiples positions qui se donnent à voir dans l'interaction des acteurs impliqués et de leurs réseaux, ancrés dans des lieux et territoires, jusqu'à l'éventuelle clôture de la controverse et de saisir la dynamique de la construction et/ou du dialogue des savoirs qui a eu lieu, ou pas. Cependant, cette partie de l'analyse ne peut se faire qu'en replaçant la situation locale étudiée dans le contexte épistémique et sociétal global. Cela peut prendre la forme d'un relevé et d'une caractérisation d'énoncés à relier à des prises de positions d'acteurs qu'il conviendra de « situer » théoriquement au regard de ce que l'on comprend de leur vision du monde et de leur rapport à la connaissance. Il est aussi souhaitable d'analyser 
la manière dont la production du discours est contrôlée et redistribuée par certains acteurs.

L'articulation entre objectifs éducatifs de court et long terme semble être une condition essentielle pour pérenniser l'implication des acteurs dans la mise en œuvre de changements attendus. Breiting (2009) rappelle l'importance d'une telle articulation, à plus forte raison dans la perspective d'un développement durable, souvent espéré de manière incantatoire comme l'indique Bray (2010). Boutinet (1995) et Villemagne (2008) montrent que l'adulte a besoin d'un réinvestissement rapide et concret dans son quotidien. Dans le même temps, il soumet son engagement à une quête de sens qui repose sur son expérience de vie, lui permettant souvent de relativiser l'impératif du court-terme, exigence qui est au cœur de l'article de Walter (2012). Il s'agit ici de favoriser auprès des acteurs la construction et surtout l'articulation signifiante et contextualisée d'identités plurielles, individuelles et collectives, sociopolitiques et culturelles mais surtout socioécologiques par la conscientisation, l'action et la mobilisation autour des questions environnementales.

Le second commentaire revient sur la question de l'implication de l'apprenant adulte. Les trois articles interpellent des logiques éducatives descendantes. Elles reposent sur des normes, des procédures et des pratiques discursives qui mobilisent l'expertise scientifique, faisant peu de cas des savoirs environnementaux portés par les acteurs locaux, experts de leurs milieux de vie. Comme le rappelle Villemagne (2008), il ne suffit pas d'informer les adultes sur les questions environnementales pour que ces derniers veuillent nécessairement prendre part à ces questions et s'engager dans des changements réfléchis et concrets. Un tel constat est largement partagé dans la communauté des chercheurs en EAE, mais ne lui est pas spécifique. Breiting (2009) par exemple, rappelle les limites d'approches qui s'annoncent comme participatives, mais qui restent formelles, centrées sur le changement de comportements et de gestes des participants, face à des approches plus ambitieuses davantage centrées sur le pouvoiragir (empowerment approach). En guise de réponse aux défis qui se présentent à l'éducation environnementale, Breiting (2009) affirme qu'« une recherche sans une ferme conscience du potentiel et des besoins d'un pouvoir-agir, passant par un engagement démocratique conçu comme résultat de l'éducation environnementale, restera limitée dans ses apports » (trad. libre). On peut se demander si ces remarques ne s'appliquent pas dans une certaine mesure, aux situations éducatives présentées dans l'article de Golding et coll. (2009).

Le troisième commentaire soulevé par cette volonté de transformation des relations que les acteurs entretiennent avec les réalités socioécologiques porte sur l'enjeu d'une pensée critique. Un tel enjeu est commun aux trois articles repérés par cette recension. Selon une étude récente menée par Fejes et Nicoll (2013), la pédagogie critique reste l'approche théorique la plus répandue en éducation des adultes. Au moins deux des articles retenus croisent l'usage d'une posture critique, avec une nécessaire prise en compte de la diversité des contextes et des perspectives socioculturelles. L'article de Bray (2010) l'évoque de manière plus indirecte par le biais du questionnement à l'égard de la culture consumériste axée sur la croissance. Les articles de Golding et coll. (2009) et de Walter (2012) se positionnent plus clairement dans cette approche théorique. Le questionnement critique porte donc sur la participation des acteurs concernés, au premier rang desquels, les chercheurs et les éducateurs eux-mêmes. Les travaux récents tendent à mettre en valeur une " posture située » pour de tels acteurs, c'est-à- 
dire une implication réflexive des chercheurs-enseignants (Walter, 2009). Une telle posture passe par une mise au jour de leurs préconceptions et une clarification des finalités de leur recherche ou des actions éducatives entreprises. Cela soulève le classique débat sur la posture neutraliste, impartiale voire objectiviste de la science, affichée par certains chercheurs, par l'expert scientifique très fréquemment, mais également parfois, par l'éducateur lui-même.

Deux des articles s'appuient sur la figure discursive particulière de la discorde au service de postures critiques, via la construction de discours contre-hégémoniques (Walter, 2012), ou bien la critique du discours prescriptif et réducteur du développement durable (Bray, 2010). La figure discursive de la discorde se trouve mobilisée par la prise en compte des conflits de valeurs et d'intérêts qui s'affrontent. Selon Burbules et Berk (1999, p. 47), la pédagogie critique concerne notamment « les parties de systèmes de croyance et d'action qui ont des effets globaux au sein des structures de pouvoir de la société. Elle interroge ces systèmes de croyance et d'action, notamment par la question, à qui cela profite? " (traduction libre). Savoir identifier les valeurs attachées aux rôles généralement endossés par des acteurs impliqués dans des situations conflictuelles peut contribuer à éclairer les postures argumentaires, les stratégies et les intérêts divergents qui s'affrontent au sein des controverses environnementales. Et ceci, d'autant plus qu'un acteur est porteur de plusieurs rôles au service de ses intérêts parfois divergents selon les contextes comme l'indique l'article de Golding et coll. (2009).

Depuis Nietzche, on ne peut plus ignorer que la question des valeurs précède celle de la vérité. Le système de valeurs et de croyances qui structure et déclenche nos intentions commande et légitime une certaine production des faits, notamment scientifiques, qui sont porteurs d'effets de vérité dans nos sociétés modernes, à plus forte raison dans les questions environnementales.

"Analyser les légitimités revendiquées renseigne sur leur nature (historique, morale, identitaire, consensuelle) et leur incompatibilité » (Monroy, 2004, p. 33). Or, comme semble l'indiquer notre recension d'écrits, une des approches les plus dynamiques en EAE est celle de l'apprentissage contextualisé selon des perspectives socioculturelles. Une telle approche centre son attention sur une meilleure prise en compte des identités culturelles que sous-tend une diversité de valeurs souvent conflictuelles portées par les acteurs selon les contextes. Les problèmes environnementaux sont typiquement des problèmes pernicieux (wicked problems), complexes où s'entrelacent des conflits d'usage et de représentations structurés par les conflits de valeurs et de cultures au sujet des ressources communes. Des situations éducatives profitant d'une controverse environnementale et s'appuyant sur les figures discursives de la concorde et de la discorde pourraient être l'occasion de jouer des scénarios d'émancipation ou d'aliénation en figurant des engagements, des injustices, leur réparation, leur négociation, etc. Reste que pour l'article de Golding et coll. (2009), la prise en compte des conflits de valeurs, d'usage et de visions uniquement de manière descriptive limite la mise en œuvre de l'approche de l'apprentissage contextualisé selon des perspectives socioculturelles. Afin de limiter notre propos, nous n'avons pas souhaité aborder la question éthique associée aux valeurs qui s'affrontent autour d'une controverse environnementale. Cette question nous parait néanmoins fondamentale. 


\section{Conclusion}

Notre première intention était de revenir sur la dimension épistémologique de la controverse environnementale. Pour ce faire, l'ébauche d'une généalogie de la controverse envisagée comme une pratique discursive rémanente dans l'histoire occidentale a permis d'évoquer sa construction progressive dans différents contextes épistémiques structurant le dialogue des savoirs qui s'échangent et circulent dans le débat entre une multiplicité d'identités. La controverse articule deux figures discursives particulières, d'une part la figure de la concorde axée sur la recherche de consensus et d'autre part, la figure de la discorde axée sur la dynamique du conflit. Toutes deux peuvent se décliner en échanges de savoirs féconds ou stériles. Ces figures discursives recoupent un débat scientifique plus large opposant les tenants de la théorie de la démocratie délibérative et les tenants de la théorie de la démocratie agonistique. Le recours à cette articulation de figures comme cadre d'analyse contribue à appréhender les situations de controverse environnementale de manière critique et dialectique.

Une recension d'écrits récents a montré que si la controverse environnementale est un objet d'attention et de recherche en éducation environnementale, elle reste marginale dans la littérature de l'éducation des adultes relative à l'environnement. Cependant, la comparaison des articles retenus permet de dégager plusieurs éléments d'un cadre théorique porteur. Une pédagogie critique pourrait mettre à profit le cadre d'analyse des deux figures discursives de la concorde et de la discorde de manière à déconstruire certains usages du savoir au service de postures de pouvoirs et d'une mise en ordre du discours par des acteurs qui cherchent à contrôler au mieux de leurs intérêts la pratique discursive de la controverse, notamment la production et la circulation des savoirs légitimes. Un tel usage pédagogique de la controverse environnementale semble devoir s'inscrire dans la multiplicité des contextes socioculturels pour mieux s'ancrer dans les situations à étudier en collaboration avec les acteurs locaux.

L'articulation de ces deux figures dans le décodage des controverses environnementales et de leur évolution peut aider à la construction de stratégies éducatives axées sur le renforcement de la réflexivité et des compétences énonciatives des acteurs, discursives et critiques. Un tel renforcement du pouvoir-agir des acteurshabitants est susceptible de consolider l'expertise citoyenne, largement inscrite dans l'horizon démocratique comme l'indique le débat actuel entre tenants de la démocratie délibérative et tenants de la démocratie agonistique.

BIBLIOGRAPHIE

Bachelart, D. (2006). Le développement durable bouscule l'éducation à l'environnement. Territoires, 466, 8-11. 
Batellier, P., et Sauvé, L. (2011). La mobilisation des citoyens autour du gaz de schiste au Québec : les leçons à tirer. Gestion, 36(2), 49-58.

Blondiaux, L. (2008). Démocratie délibérative vs. démocratie agonistique ? Raisons politiques, 30(2), 131-147.

Bouchard, F., Guay, L., Truilhé-Marengo, E., Reber, B., et Chicoine, L. (2013). Controverses environnementales : expertise et expertise de l'expertise. [Vertigo] La revue électronique en sciences de l'environnement, 13(2). Récupéré le 29 décembre 2014 de http://vertigo.revues.org/ 13961

Bourdieu, P. (1980). Questions de sociologie. Paris : Minuit.

Boutinet, J.-P. (1995). Psychologie de la vie adulte. Paris : Presses universitaires de France.

Bray, R. (2010). The Importance of Controversy : Is Education for Sustainable Development Too Nice ? Journal of Sustainability Education (JSE). Récupéré le 29 décembre 2014 de www.jsedimensions.org/wordpress/content/the-importance-of-controversy-is-education-forsustainable-development-too-nice_2010_05/

Breiting, S. (2009). Issues for environmental education and ESD research development : looking ahead from WEEC 2007 in Durban. Environmental Education Research, 15(2), 199-207.

Burbules, N. C., et Berk, R. (1999). Critical Thinking and Critical Pedagogy : Relations, Differences, and Limits. Dans T. S. Popkewitz et L. Fendler (Éd.), Critical Theories in Education (p. 45-65). New York : Routledge.

Callon, M. (1981). Pour une sociologie des controverses technologiques. Fundamenta Scientiae, 2(3/4), 381-399.

Callon, M., Lascoumes, P. et Barthe, Y. (2001). Agir dans un monde incertain : essai sur la démocratie technique. Paris : Seuil.

Clover, D. E. (2003). Environmental adult education : Critique and creativity in a globalizing world. New Directions for Adult and Continuing Education, 2003(99), 5-15.

Fabiani, J.-L. (1997). Controverses scientifiques, controverses philosophiques. Enquête 5, 11-34.

Fejes, A. et Nicoll, K. (2013). Editorial : Approaches to research in the education and learning of adults. European journal for Research on the Education and Learning of Adults, 4(1), 7-16.

Foucault, M. (1969). L'archéologie du savoir. Paris : Gallimard.

Gil, F. (s. d.). Controverse. Encyclopædia Universalis. Consulté le 29 décembre 2013 de www.universalis.fr/encyclopedie/controverse/

Golding, B., Brown, M., Foley, A., Smith, E., Campbell, C., Schulz, C., Grace, L. (2009).

Wicked Learning : Reflecting on « Learning to Be Drier ». Australian Journal of Adult Learning, 49(3), 544-566.

Larochelle, M. et Désautels, J. (2006). L'éducation aux sciences et le croisement des expertises.

Dans A. Legardez, et L. Simonneaux, L'école à l'épreuve de l'actualité. Enseigner les questions vives (p. 61-77). Paris : ESF.

Limoges, C., Doray, P., Henrichon, P., Cimon, M., Veilleux, D., Charron, D., et Davignon, L. (1993). L'Etat et les pre occupations des citoyens relatives aux incidences du changement technologique : la regulation publique en contexte d'« environnementalisation ». Sainte-Foy, Quebec : Gouvernement du Quebec, Conseil de la science et de la technologie. 
Monroy, M. (2004). L’engrenage conflictuel. Éducation \& management, (26), 30-33.

Sauvé, L. (2007). L'équivoque du développement durable. Chemin de traverse, 4, 31-47.

Thomashow, M. (1995). Ecological Identity : Becoming a Reflective Environmentalist. Cambridge, Mass : The MIT Press.

Vernant, J.-P. et Vidal-Naquet, P. (2001). Mythe et tragédie en Grèce ancienne. II. Paris : La Découverte.

Villemagne, C. (2008). Regard sur l'éducation relative à l'environnement des adultes. [VertigO] La revue électronique en sciences de l'environnement, 8(1). Récupéré le 29 décembre 2013 de http://vertigo.revues.org/1915

Walter, P. (2009). Philosophies of Adult Environmental Education. Adult Education Quarterly : A Journal of Research and Theory, 60(1), 3-25.

Walter, P. (2012). Cultural Codes as Catalysts for Collective Conscientisation in Environmental Adult Education : Mr. Floatie, Tree Squatting and Save-Our-Surfers. Australian Journal of Adult Learning, 52(1), 114-133.

Welsh, R. (1993). Education and Action for the Urban Environment. Adults Learning (England), $4(8), 212-13$.

Yannick, A., Kervarec, F., et Verena, M. (2009). Gestion concertée de l'environnement : Itinéraire vers des engagements volontaires forcés. [Vertigo] La revue électronique en sciences de l'environnement, 9(1). Récupéré le 29 décembre 2014 de http://vertigo.revues.org/8566

\section{NOTES}

1. Suivant Foucault (1969), une pratique discursive est à la fois une forme d'action de type praxique ou pragmatique mais aussi un ensemble de règles anonymes historiques et contextualisées déterminant les conditions d'exercice de la parole, notamment les conditions selon lesquelles telle parole est légitime ou pas, ce qui participe d'une mise en ordre du discours.

2. Cette appellation permet de garder le sigle (EAE) utilisé en langue anglaise pour Environmental Adult Education

3. Cette esquisse de généalogie se limite à la culture occidentale. Il est probable qu'une telle pratique discursive donnant lieu à une forme de construction du savoir se retrouve dans d'autres contextes socio-culturels.

4. Remerciements à Pierre Doray pour ses conseils éclairés et la référence à ce rapport dont il est l'un des auteurs.

5. Dans le théâtre grec, elle est associée au masque porté par l'acteur, figure toujours dialectique à deux faces complémentaires, traduisant les effets de tensions en termes contraires (terreur et grotesque, réalité et illusion, sauvagerie et culture, etc.). Ces rôles dialectiques favorisent un lent apprentissage des différences et permettent au futur citoyen une bonne intégration dans la vie civique (Vernant et Vidal-Naquet, 2001).

6. Les bases de données utilisées sont Francis, Repère et Érudit pour les revues francophones et CBCA, Scopus, Eric pour les revues anglophones. Pour la présente recension, onze articles ont été repérés dont deux en français. Au final, trois articles, tous anglophones, ont été retenus sur la période 2009-2013. Ils abordent la controverse environnementale indirectement. En effet, aucun article ne cible la controverse environnementale ou les questions sociotechniques ou socioécologiques comme objet didactique en éducation des adultes non-formelle et informelle. 
7. Sur les onze articles repérés, sept étaient plutôt campés en éducation formelle, dont trois articles francophones, mais la plupart n'abordaient pas la controverse environnementale comme sujet central du propos.

\section{RÉSUMÉS}

La controverse publique s'est imposée comme une forme récurrente de la dynamique démocratique. La montée des préoccupations environnementales a conforté cette pratique discursive, qui mobilise différents types de savoirs. Appréhendée par plusieurs disciplines académiques, la controverse environnementale reste encore peu abordée en éducation des adultes relative à l'environnement (EAE). Par ailleurs, le consensus reste la visée ultime sans que les potentialités du conflit ne soient toujours pleinement exploitées dans une perspective critique, pourtant dominante en éducation des adultes. À partir d'une exploration de la littérature récente en $\mathrm{EAE}$, cet article esquisse une généalogie de la controverse envisagée selon deux modalités discursives de construction du savoir repérables que j'appellerai figure de la discorde et figure de la concorde, afin de rendre compte des usages du consensus et du conflit et leurs apports potentiels pour la construction d'une grille d'analyse des controverses environnementales.

Public controversy has become a recurrent form of democratic dynamics. The rise of environmental concerns has consolidated this discursive practice, which mobilizes various types of knowledge. Addressed by several academic disciplines, environmental controversy remains under-explored within the field of environmental adult education (EAE). Moreover, the consensus remains the ultimate goal without the potential of conflict is not fully exploited from a critical perspective, yet dominant in adult education. By exploring the recent literature in EAE, this article outlines a genealogy of environmental controversy as considered by two discursive modalities of knowledge construction of the knowledge. I call them figure of discord and figure of concord, to reflect the uses of consensus and conflict and their potential contributions to the construction of an analysis grid for environmental controversies.

\section{INDEX}

Mots-clés : controverse, conflit, consensus, savoirs critiques, politique, éducation des adultes

Keywords : controversy, conflict, consensus, critical knowledge, politics, adult education

\section{AUTEUR}

\section{JÉRÔME LAFITTE}

Doctorant en cotutelle sous la direction de Lucie Sauvé au Centre de recherche en éducation et formation relatives à l'environnement et à l'écocitoyenneté de l'Université de Québec à Montréal, et sous la direction de Christine Vergnolle-Mainar au laboratoire de géographie Géode de l'université de Toulouse 2 - Jean Jaurès. Sa recherche doctorale explore les relations entre 
temporalités environnementales et la dialogique des savoirs au sein de territoires en projets de « développement durable». 